\title{
Strates
}

STRATES Matériaux pour la recherche en sciences sociales

$12 \mid 2006$

Nouvelles tensions impériales et recompositions en

Europe centrale, orientale, et CEI

\section{Ethnicité et « renaissantisme » ethnique chez les peuples autochtones de la République sakha (Yakoutie)}

\author{
Marine Le Berre-Semenov
}

\section{CpenEdition}

Journals

Édition électronique

URL : http://journals.openedition.org/strates/1882

ISSN : $1777-5442$

Éditeur

Laboratoire Ladyss

Édition imprimée

Date de publication : 31 décembre 2006

ISSN : 0768-8067

Référence électronique

Marine Le Berre-Semenov, «Ethnicité et « renaissantisme » ethnique chez les peuples autochtones de la République sakha (Yakoutie) », Strates [En ligne], 12 | 2006, mis en ligne le 19 juillet 2007, consulté le 07 septembre 2020. URL : http://journals.openedition.org/strates/1882

Ce document a été généré automatiquement le 7 septembre 2020

Tous droits réservés 


\title{
Ethnicité et « renaissantisme » ethnique chez les peuples autochtones de la République sakha (Yakoutie)
}

\author{
Marine Le Berre-Semenov
}

1 La Yakoutie se situe dans le Nord-Est de la Russie : c'est une région très étendue, près de trois millions de $\mathrm{km}^{2}$, et très peu peuplée en raison de ses conditions climatiques extrêmes, environ un million d'individus au total. Cette population se compose pour plus de la moitié d'allochtones, principalement des Russes, mais aussi des Biélorusses, Ukrainiens, Tatars, Bouriates, et à peu près de toutes les nationalités de l'ex-URSS. La présence de ces allochtones est liée pour l'essentiel à l'exploitation des ressources naturelles, nombreuses et abondantes, diamants, or, gaz, pétrole, bois, etc. Le reste de la population est autochtone : les Yakoutes ou Sakhas, c'est-à-dire l'ethnie titulaire, regroupent environ 365000 individus $^{1}$ concentrés pour l'essentiel dans les régions centrales, sur le cours moyen de la Léna et sur le Viljuj. La population autochtone comprend encore plusieurs ethnies minoritaires, réparties essentiellement à la périphérie du territoire: il s'agit des Evenks, des Evènes, des Youkaguirs, des Tchouktches et des Dolganes. Pris tous ensemble, ces groupes ne rassemblent pas plus de 25000 individus. Les peuples autochtones de la Yakoutie se rattachent à ce que l'on nomme la "civilisation circumpolaire ", ou à ce que l'on peut également nommer la "sociodiversité boréale » : ce sont traditionnellement des peuples nomades ou seminomades, vivant de la chasse, de la pêche, de la cueillette, et surtout, pour les minorités, de l'élevage du renne, et pour les Sakhas, de l'élevage du bétail et notamment du cheval. Ce sont enfin des peuples qui ont en commun une tradition chamaniste et animiste.

2 La Yakoutie est née, comme république ou entité stato-nationale, en 1922, à une époque où l'Union soviétique naissante réorganisait la division territoriale et administrative de ce qui avait été l'Empire russe. Soixante-dix ans plus tard, peu de temps avant que 
l'URSS n'éclate à son tour, en 1990, la Yakoutie participe à un mouvement général qualifié de "parade des souverainetés ", et déclare sa souveraineté à l'intérieur de la Fédération de Russie. À cette époque, les élites intellectuelles et politiques de la Yakoutie voient dans la souveraineté l'unique moyen de garantir la sauvegarde et la «renaissance» des peuples autochtones. L'idée de renaissance s'oppose à celles de déclin et d'extinction. Sur quoi repose la problématique de déclin ou d'extinction des peuples autochtones en Yakoutie, et comment ceux-ci s'organisent-ils dans la période post-soviétique pour conjurer cette menace, qu'elle soit réelle ou fantasmatique?

Causes et origines de la problématique d'extinction des peuples du Nord

3 L'extinction des indigènes sibériens est une problématique ancienne. Au XIX siècle, ces peuples connaissent une situation démographique et sanitaire catastrophique qui est interprétée par les représentants du pouvoir colonial comme la preuve d'une arriération culturelle et même d'une infériorité biologique des indigènes sibériens, et par les opposants au régime tsariste, comme la conséquence essentielle de leur exploitation socio-économique. C'est à ces derniers, les révolutionnaires, que l'on doit la naissance du concept ou de l'idée de renaissance dès la fin du XIx $x^{e}$ siècle, concept qui sera ensuite repris et approfondi par les premiers intellectuels autochtones et par leurs héritiers contemporains. Au tout début de la période soviétique, sous l'influence de cette idée, les peuples du Nord bénéficient d'une politique de protection et de discrimination positive ; elle va cependant très vite être remplacée par la politique de soviétisation qui vise à fondre toutes les ethnies, et en premier lieu les plus minoritaires, dans la matrice du nouveau peuple soviétique. En Yakoutie, cette politique a emprunté des voies différentes selon les peuples : radicales et déclarées pour les ethnies minoritaires, plus progressives et larvées pour les Yakoutes. Les méthodes utilisées étaient cependant les mêmes: le déracinement géographique imposé avec le regroupement des populations dans des bourgades où le mélange des clans, des classes et des ethnies est programmé ; l'éducation soviétique en langue russe, ou dans une langue yakoute volontairement russifiée; la diabolisation du sentiment ethnique; la folklorisation des cultures traditionnelles.

4 Dès la fin de la période soviétique, et plus encore après la chute du régime, les Yakoutes et les ethnies minoritaires vont subir le contrecoup de toutes les transformations radicales qui leur ont été imposées par le pouvoir soviétique. Dans le nouveau contexte, marqué par le passage brutal et douloureux à un nouveau modèle de société, le sentiment d'extinction collective ressurgit chez les peuples du Nord. Ce sentiment est désormais lié, par des segments de plus en plus larges de la population, à la perte des caractéristiques qui faisaient exister en son sein des entités sociales et culturelles intégrées et autonomes: les modes de vie et de pensée, les structures sociales, les traditions, les langues vernaculaires - toutes les composantes des sociétés autochtones se désagrègent rapidement et sombrent peu à peu dans l'oubli. Le sentiment d'extinction collective est lié, encore et surtout, aux nombreuses conséquences de tous ces changements, porteuses de destructions en tout genre que l'on peut désormais dénoncer plus librement comme étant en grande partie liées aux méthodes économiques outrancières et destructrices du régime soviétique; la dégradation générale des indicateurs démographiques et humains chez les peuples du Nord dans la période contemporaine résulte en effet de la destruction des équilibres sociaux et écologiques traditionnels, et de la déculturation provoquée par la tentative de soviétisation forcée. 
5 Les mouvements et les stratégies de renaissance qui se mettent en place dans la période post-soviétique traduisent d'abord la volonté des élites autochtones de renverser la situation de dépérissement ou de déclin dans laquelle se trouvent leurs peuples, et de les amener peu à peu vers une renaissance. Ces mouvements s'apparentent, comme nous allons le voir, à des tentatives de reconstruction des ethnies autochtones sur le plan identitaire, social, culturel, religieux et linguistique, ce qui pose bien entendu la question de l'ethnie, et encore plus celle de l'ethnicité, de ses manifestations et de ses variations sous l'influence des acteurs sociaux et politiques.

Ethnie, ethnicité : écarts de conception

6 Dans le cas des peuples autochtones de la Yakoutie, les acteurs locaux ont tendance à occulter le caractère éminemment subjectif, relatif et perpétuellement mouvant de l'ethnicité. Même si ce caractère se dégage avec force de l'histoire de la colonisation de la Yakoutie, car si l'on étudie cette histoire, on voit bien, en effet, que les noms de «Dolganes", "Youkaguirs", "Yakoutes », etc., s'attachent à des catégories plus ou moins abstraites, construites, déconstruites, puis reconstruites et institutionnalisées au gré des colonisations successives, russe et soviétique, et, aujourd'hui, de la décolonisation, si tant est que l'on puisse considérer la souveraineté comme un début de décolonisation. Ces catégories ethniques sont le fruit de la présence et des politiques $\mathrm{du}$ colonisateur, lequel, pour asseoir sa domination politique, économique et culturelle sur les différentes populations de la Yakoutie, a suscité l'apparition de nouvelles divisions au sein des sociétés locales, divisions territoriales, socioculturelles, socioéconomiques et, finalement, ethniques. Les flux de population occasionnés par la colonisation et ses différentes politiques ont eux aussi largement contribué, au fil du temps, à faire décliner et parfois disparaître certaines cultures, comme ils ont contribué à faire naître et à développer de nouvelles identités locales, dont certaines ont même été officialisées par un acte d'ethnicisation administrative, c'est le cas par exemple des Dolganes.

7 L'occultation de cet aspect très important, quasi central, de l'ethnicité par les acteurs des mouvements de renaissance en Yakoutie découle d'une conception particulière de l'ethnie, très répandue en Russie dans la période post-soviétique, comme d'un organisme biosocial. Cette conception hybride car elle se nourrit de thèses de personnes aussi différentes que Joseph Staline et Lev Gumilev dont les thèses connaissent alors un succès sans précédent, influence très largement la couleur et l'orientation des mouvements de renaissance autochtone en Yakoutie : dans l'esprit des renaissantistes, les ethnies correspondant de moins en moins à ce qu'elles devraient être selon la représentation qu'ils en ont, l'urgence est de les rendre « conformes » ou "normales", et ainsi les sauver de l'extinction, puisque aucune autre existence collective ne semble désormais admise en-dehors de l'ethnie. Ceci explique que l'une des grandes orientations communes aux différents renaissantismes en Yakoutie soit l'ethnicisation, ce que nous allons essayer de mettre en évidence d'abord pour les Sakhas, puis pour les ethnies minoritaires.

Renaissantisme des Sakhas

8 Pour les Sakhas, la renaissance est d'abord un ensemble d'aspirations qui commencent à s'exprimer à partir de la Perestroika : il y a la volonté de s'extraire d'une situation d'oppression conditionnée par la politique de soviétisation et par la minorisation globale (numérique, économique, culturelle, psychologique) de l'ethnie titulaire par des migrants allochtones présents pour l'exploitation industrielle des richesses naturelles 
de la Yakoutie; il y a également l'envie de se réapproprier l'identité locale, traditionnelle, mise en péril par l'identité dominante et imposée de la nation russosoviétique ; enfin, il y a l'espoir de retrouver une certaine autonomie de développement social, culturel, économique, qui, seule, garantirait durablement la renaissance des Sakhas.

La souveraineté proclamée par la Yakoutie en 1990 marque le début d'une nouvelle ère de construction "stato-nationale » et de "yakoutisation »: les élites yakoutes ne font là que reprendre l'œuvre de leurs prédécesseurs, tous supprimés par la répression politique dans les années 1930 et 1940. La renaissance du peuple sakha est conceptualisée et popularisée par l'intelligentsia nationale, elle est aussi prônée et soutenue, dans ses aspects culturels et éducatifs surtout, par l'État et les institutions de la Yakoutie souveraine. Dans ce cadre a priori favorable à leur cause, les renaissantistes yakoutes s'efforcent de libérer les consciences de leurs contemporains par un travail de mémoire sur les périodes sombres et les injustices de l'histoire coloniale. Ils s'efforcent également de reconstruire les éléments fondateurs d'une identité sakha : à travers des discours, des productions littéraires, scientifiques, artistiques et des manifestations diverses, ils cherchent à redonner aux Sakhas une image valorisante de leur ethnicité. Ils essayent aussi de développer les fonctions de la langue sakha et de restaurer (au besoin en les réinventant) les traditions orales, esthétiques, religieuses, sportives, etc., dans la pratique des individus.

L'objectif des élites yakoutes est finalement d'assurer, par la désoviétisation et la reethnicisation des masses, le maintien du peuple sakha et le développement de sa culture, de sa langue et de sa conscience ethnique. L'ethnicisation des Sakhas, ou la yakoutisation, peut finalement se comprendre comme la tentative d'éradiquer les conséquences de la présence russe en Yakoutie, devenue massive au cours de la seconde moitié du $\mathrm{xx}^{\mathrm{e}}$ siècle. L'affirmation identitaire des Sakhas face à la majorité russophone est l'un des principaux moteurs du renaissantisme sakha, c'est en effet dans cette relation que se trouve la principale source des tensions socio-identitaires en Yakoutie : d'une part, entre les Sakhas et les Russes, considérés comme une population d'occupation, d'autre part, entre les Sakhas ruraux, gardiens de l'identité traditionnelle, et les Yakoutes urbanisés, en grande partie assimilés à la culture russosoviétique.

Renaissantismes des ethnies minoritaires (Evenks, Evènes, Dolganes, Youkaguirs, Tchouktches)

Globalement, la mobilisation renaissantiste des leaders des minorités autochtones en Yakoutie se présente comme un vaste effort de reconstruction identitaire et sociale des groupes évenk, évène, youkaguire, dolgane et tchouktche. Cette reconstruction se fait sur la base de réformes et de mesures d'ethnicisation, de retour aux traditions et de mesures de modernisation, dont l'application reste cependant, pour de multiples raisons, disparate et limitée. Là aussi, l'ethnicisation est l'un des aspects fondamentaux de ce mouvement de renaissance des ethnies minoritaires. Elle se réalise essentiellement par le biais des "écoles nationales ", où les travaux des intellectuels sur l'histoire ethnique, la langue et la culture locales sont enseignés aux élèves des minorités. Elle se réalise encore au travers de fêtes, de congrès et d'autres manifestations ethniques, qui sont autant d'occasion de réinventer l'ethnicité perdue ou déclinante des autochtones. Cette ethnicisation a, comme nous avons pu le 
constater, des effets contrastés, à la fois positifs et négatifs, sur la vie des communautés autochtones :

- dans la période post-soviétique, les ethnies minoritaires du Nord sont touchées de plein fouet par la paupérisation. L'élément décisif du dépérissement des communautés autochtones dans la période nouvelle est néanmoins la déculturation, c'est-à-dire, la perte de tout ce qui permettait aux individus de se construire comme des êtres à part entière: liens familiaux et sociaux, moyens d'existence, mémoire collective, langue maternelle, spiritualité, valeurs, etc. Face au problème de la déculturation des minorités autochtones, au sein desquelles nombre d'individus semblent ne plus savoir ni «comment» ni «pourquoi » vivre, l'ethnicisation dans ses aspects à la fois symboliques et pratiques - réapprendre les traditions d'existence au sein de la nature boréale, renouer le lien avec la terre et les ancêtres - présente une voie de renaissance très plausible ;

- en même temps, l'ethnicisation impose aux autochtones de s'identifier à des cadres qui n'étaient déjà pas forcément pertinents lors de leur création, et qui, s'ils l'étaient ou le sont devenus, ont progressivement cessé de l'être au gré de la politique d'assimilation. L'ethnicisation représente, en outre, une source potentielle de dissension pour des populations très mélangées et un facteur supplémentaire d'aliénation pour les autochtones assimilés ou métissés à qui elle impose un modèle souvent trop éloigné de leur identité propre.

14 Au niveau microsocial, enfin, les autochtones semblent aspirer à une renaissance, que l'on ne saurait qualifier d'ethnique : ceux-ci souhaitent avant tout rester sur leurs terres ancestrales, et perpétuer les activités qui sont au cœur de leurs cultures et de leurs identités - la renniculture, la chasse, la pêche. C'est ce que traduit l'élaboration de stratégies de sauvegarde de la vie nomade et des activités traditionnelles, ou de projets plus élaborés d'autogestion locale, dont l'objectif est finalement de permettre le retour des communautés à une certaine autonomie de développement. Cette quête d'autonomie répond au besoin vital de se protéger des aléas de l'histoire et des caprices de la modernité, puis à une nécessité objective - mais peu explicitée - de reconstruire des liens primordiaux, liens familiaux et liens sociaux, laminés par l'expérience soviétique.

15 Pour compléter ce tableau, il convient maintenant de revenir plus en détail sur la question du mode de pensée et d'analyse des acteurs renaissantistes.

Modes de pensée et d'analyse des renaissantistes

Si, dans la pratique, les renaissantismes autochtones en Yakoutie se présentent comme des tentatives complexes et multidimensionnelles de reconstruction des ethnies, en revanche, dans l'esprit des renaissantistes, la renaissance s'apparente bel et bien à un processus orienté de revitalisation sociobiologique ou ethnogénétique des peuples, dans le droit fil de la pensée gumilovienne. Pour les renaissantistes, tout homme est le représentant d'une ethnie dont il doit possèder la culture et toutes les marques d'ethnicité requises, cette appartenance n'étant pas liée à un choix, mais déterminée par le sang. L'idée que le déclin des ethnies, outre ses causes sociales, politiques et économiques, est aussi un problème à la fois de pureté du sang et de santé des gènes occupe une place importante dans ces mouvements de renaissance. D'une manière générale, la confusion entre les notions sociales et les notions biologiques ou génétiques est omniprésente dans les analyses des renaissantistes qui ont souvent recours à des théories douteuses, sur le plan scientifique et parfois éthique : il existe 
par exemple un concept de "mémoire génétique ", selon lequel chaque individu aurait dans ses gènes l'instinct ou l'intuition de sa culture d'origine. La récurrence de ce type d'analyses trouve différentes explications : d'une part, il y a là un moyen d'envisager la renaissance des peuples autochtones, quelle que soit leur situation sur le plan de l'assimilation et du métissage. D'autre part, l'obsession des gènes traduit aussi la vive inquiétude suscitée par certains problèmes bel et bien cruciaux des populations autochtones : au centre de ces problèmes, il y a l'alcoolisme ravageur, et non seulement ses causes (le bouleversement des modes de vie, la perte des traditions, la destruction des équilibres sociaux et écologiques, l'assimilation, etc.), mais, encore plus, ses conséquences sur la situation démographique des populations autochtones, et sur la santé mentale et physique des nouvelles générations. Enfin, les développements sociobiologiques des renaissantistes résultent vraisemblablement de la tendance qu'ont ces derniers à analyser tous ces problèmes propres à leurs peuples, et qui sont aussi leurs propres problèmes, d'une manière qui n'est pas scientifique, mais fortement guidée par des angoisses, des complexes et des fantasmes personnels, idéologisés dans les théories en circulation dans l'ensemble post-soviétique.

17 Face à ce constat, on doit s'interroger sur l'incidence que peut avoir sur les processus sociétaux une propagande basée sur ce type de conceptions. Le renaissantisme en Yakoutie, et ses conceptions «ethnogénéticiennes en particulier, peuvent être considérés comme une nouvelle idéologie institutionnelle, née en réaction à l'idéologie soviétique ». Ainsi, en même temps qu'ils prônent la réappropriation volontaire par les individus des critères d'ethnicité attachés à leur communauté d'origine, les renaissantistes déconseillent ouvertement de fonder un foyer avec une personne d'un autre sang. D'ores et déjà, on peut dire que les renaissantistes ont une influence perceptible sur les processus sociétaux, et donc une incidence concrète sur la vie des populations : cette influence comporte des aspects à la fois positifs et négatifs. Parmi les aspects positifs, on peut citer la déconstruction des complexes et des tabous identitaires légués par la colonisation, très importante et parfaitement pertinente dans un processus de renaissance. En même temps, en propageant l'idée que tout « homme véritable" ne peut et ne doit être qu'un "homo-ethnicus", les renaissantistes contribuent à faire naître de nouveaux complexes et tabous identitaires chez leurs contemporains : si l'on ne taxe plus les individus attachés à leur culture ethnique de nationalisme, en revanche, dans la première décennie post-soviétique, on pointe du doigt les «nihilistes nationaux » ou les métis, dits "marginaux », qui contrarient la vision d'une humanité scindée en familles ethniques bien dissociées les unes des autres.

Pour conclure, les manipulations de l'ethnicité auxquelles se livrent les élites autochtones en Yakoutie dans la période de la Souveraineté ${ }^{2}$ ont pour but de faire renaître des ethnies confrontées à un dépérissement et à un déclin à la fois socioéconomique, démographique et culturel. Cependant, le traitement pseudo-scientifique de la question de l'ethnicité et l'exploitation idéologique qui en est faite par les mêmes acteurs sociaux et politiques tendent à induire de nouveaux critères d'exclusion et de division au sein des communautés autochtones, qui ont des incidences négatives sur les relations interethniques et sont sources de tensions nouvelles, notamment entre les Russes et les autochtones, et les différentes minorités entre elles. Cette situation étant une conséquence directe de l'histoire coloniale russe et soviétique, l'analyse des phénomènes observés dans cette région de la Fédération de Russie est applicable aussi à 
d'autres régions nationales de l'ex-URSS, et en premier lieu aux régions de la Sibérie, du Nord et de l'Extrême-Orient russe.

\section{NOTES}

1. D’après les données du recensement de 1989.

2. On peut considérer que la période de la Souveraineté en Yakoutie correspond aux deux mandats du premier président yakoute, M. Nikolaev, entre 1990 et 2001. En effet, son successeur, élu en avril 2001, V. Shtyrov, mène une politique plus " russienne », conforme aux attentes et exigences de V. Poutine.

\section{RÉSUMÉS}

M. Leberre Semenov analyse la stratégie identitaire ethniciste des élites sahkas qui ont essayé par ce biais durant les quinze dernières années d'asseoir leur pouvoir politique et culturel sur leur territoire, pour s'en voir récemment dépossédées par la révision politique préconisée par Vladimir Poutine, désireux de contrôler les richesses minières considérables de leur république. Selon cet auteur, cette stratégie «identitaire» a créé de nouvelles divisions difficiles à surmonter.

Marine Le Berre Semenov. Born-again etnicity of small people from Sakha republic (Yakoutia)

M. le Berre Semenov analyses closely the "born-again ethnicist" identity strategy of the Sakha elites, who have tried by this means to anchor their cultural and political power in their territory during the last fifteen years, only to lose it recently through the political revision recommended by Vladimir Putin in his desire to control their republics' considerable mining wealth. In her opinion, this "identity" strategy has caused new and difficult divisions.

\section{AUTEUR}

\section{MARINE LE BERRE-SEMENOV}

Docteur de l'Inalco, membre du CRREA (Centre de recherches russes et euro-asiatiques), marine.sem@free.fr 